\title{
Conducta sexual y riesgo de embarazo temprano en adolescentes de Cd. Victoria, Tamaulipas
}

\author{
Sexuality and practices of risk among \\ adolescents in CD. Victora Tamps.
}

M.Ed. Olga Lidia Banda González • MCS. Ma. Ramona Medrano Martínez;• MCS.

Cinthya Patricia Ibarra González; MCE. Ma. Guadalupe Vázquez Salazar;•

MCE . Guadalupe Esmeralda Vázquez Treviño.

\section{Resumen}

Introducción: La proporción de adolescentes que han iniciado relaciones sexuales va en aumento y la edad de inicio esta disminuyendo, sin embargo la capacidad para evitar el embarazo no acompaña este comportamiento. En la Jurisdicción Sanitaria No. 1 de Cd. Victoria, Tamaulipas, en el año 2008 se atendieron 15,137 embarazadas, y de estas el 22.0\% son menores de 20 años,1 afectándoles de manera importante los planes de vida y futuro de las madres adolescentes. El propósito de esta investigación fue describir las características de la conducta sexual de los adolescentes, variables que se relacionan con el inicio de la actividad sexual, y practicas de riesgo para embarazo. Metodología: Se realizo un estudio observacional, descriptivo, transversal, correlacional.2. En 1212 adolescentes de 12 a 19 años ambos sexos, mediante un muestreo no probabilístico, por disponibilidad. Utilizando un instrumento con un alpha de cronbach de 0.82. Resultados. La mitad de los participantes en el estudio se encuentra en el rango de 15 a 17 años con una media de 16 años. El 97\% conoce métodos anticonceptivos, mas no su funcionamiento la información la adquirieron de la escuela y amigos. El $22 \%$ tiene vida sexual activa iniciando entre los 14-15 años, no utilizaron método anticonceptivo por no planear la relación sexual. El 50\% tiene más de una pareja, no se encontró asociación entre la edad y el número de parejas ni entre la edad y la última vez en que se tuvo relación sexual. Discusión: Los datos obtenidos sobre conducta sexual no difieren con lo reportado en la revisión de la literatura, la actividad sexual inicia más tempranamente entre los hombres que en las mujeres, el uso de métodos anticonceptivos no es generalizable ni en la primera ni en la

-Profesora de Carrera Asociado B TC en la ENEO-UNAM.

••Alumnas del octavo semestre de la carrera de Licenciatura en Enfermería y Obstetricia en la ENEO-UNAM.

Correspondencia: Martha lilia Bernal Becerril • marthaliliabb@hotmail.com

RECIBIDO: 10 DE NOVIEMBRE DE 2009.

ENVIADO A CORRECIONES: 7 DE DICIEMBRE DE 2009

ACEPTADO: 8 DE ENERO DE 2010 
última relación sexual. Conclusiones: Un porcentaje alto no tiene conocimiento sobre métodos anticonceptivos y su funcionamiento, considerándose estas variables como riesgo para la presentación de embarazo en la adolescencia.

\section{Abtract}

Introduction: The proportion of adolescents who have initiated sexual relations is increasing and they are beginning at an earlier age, nevertheless the ability to avoid pregnancy does not accompany this behavior. At the Sanitary Jurisdiction number 1,in Cd. Victoria, Tamaulipas; year 2008. they took care of 15,137 pregnant women, and of these, $22.0 \%$ are pregnancies in subjects who are younger than 20 years, affecting them in a considerable way in relation to life plans and the future of the adolescent mothers. Objetive: The aim of this research was to describe the characteristics of the adolescent sexual behavior, variables related to the beginning of sexual activity and risk practices for pregnancy. Methodology: A correlational, transversal, descriptive and observational study was carried out (Polit \& Hungler, 1999) in adolescents of 12 to 19 years of age, both sexes, through a non-probabilistic sampling by availability, using an instrument with a Cronbach's alpha of 0.82 Results: The sampling was of 1212, half of the cases are in the rank of 15 to 17 years and the mean is 16 years. 97\% know about contraceptive methods but they do not know how they work. The information was acquired in school and from friends. 22\% have active sexual life beginning between 14 and 15 years. They did not use a contraceptive method because the sexual relation was not planned. $50 \%$ have more than one couple. There was no association between age and the number of couples or between the age and the last time that they had sexual relations. Discussion: The information that we obtained about sexual behavior do not differ from the one that was reported on the literature revision. Sexual activity begins earlier in men than in women. The use of contraceptive methods is not generalizable, neither in the first nor in the last sexual relation. Conclusions: A high percentage do not know about contraceptive methods and how they work, considering these variables as a risk of pregnancy in adolescents.

\section{INTRODUCCIÓN.}

La adolescencia (10 a los 19 años de edad $)^{3}$ es la etapa entre la niñez y la edad adulta, constituye una de las etapas más vulnerables del ser humano para desarrollar costumbres y hábitos de riesgo para su salud, por la diversidad de cambios relacionados con el crecimiento, independencia y maduración.

En este ciclo vital se presentan cambios tanto en el aspecto fisioló- gico (estimulación y funcionamiento de los órganos por hormonas, femeninas y masculinas), cambios estructurales anatómicos y modificación en el perfil psicológico y de la personalidad; sin embargo la condición de la adolescencia no es uniforme y varía de acuerdo a las características individuales y de grupo.

Cada individuo posee una personalidad y en esta etapa es cuando más la manifiesta, no solo de manera individual sino de forma grupal, para poder medir el grado de aceptación en los diferentes escenarios sociales e ir haciendo los ajustes o modificaciones necesarios para sentirse aceptado socialmente.

El perfil psicológico generalmente es transitorio y emocionalmente inestable. El desarrollo de la personalidad dependerá en gran 
medida de los aspectos hereditarios, de la estructura y experiencias en la etapa infantil preescolar y escolar y de las condiciones sociales, familiares y ambientales en el que se desenvuelva el adolescente.

Por la misma inestabilidad emocional, la búsqueda de identidad y de su proyectos de vida, interés por lo prohibido y la rebeldía contra lo establecido, la influencia de compañeros, cultura permisiva, los medios de comunicación, el deseo de experimentar una nueva vivencia, y el desconocimiento de métodos anticonceptivos, son factores que los exponen en situaciones desfavorables y en riesgo de modificarles sustancialmente la calidad y expectativas de vida, por la presencia de un embarazo, afectando al núcleo familiar y a la sociedad en general.

El embarazo no planeado en esta etapa de la vida, se asocia con el inicio temprano de relaciones sexuales y el uso inconsistente de métodos anticonceptivos, incluyendo el condón ${ }^{4}$ esta situación puede modificarse mediante una educación sexual integral como base en el comportamiento de una sexualidad segura, satisfactoria, responsable y libre de riesgos, para lograrlo es indispensable informar a los adolescentes de manera clara y objetiva sobre salud sexual y reproductiva.

Existen estudios que muestran que la práctica de sexo no protegido sigue siendo el factor mas importante para adquirir VIH/SIDA y embarazos no planeados 5 , por lo que es importante además de educar, fomentar los valores que les permitan resistir la tentación de iniciar la actividad sexual a temprana edad sin protección. En México, se estima que la edad de la primera relación sexual se ubica alrededor de los 16 años ${ }^{4}$.

En relación a esta temática González-Garza y cols ${ }^{6}$ realizaron un estudio con el propósito de describir las características del comportamiento sexual de los adolescentes de 12 a 19 años, su grado de conocimiento sobre anticoncepción, las variables que se relacionan con la utilización de anticonceptivos en la primera relación sexual y las que se asocian con el embarazo. Se analizo la base de datos de adolescentes de la Encuesta Nacional de Salud 2000.

Dentro de los hallazgos más importantes el $69.2 \%$ refirió conocer al menos un método de control de la fecundidad, incrementándose el porcentaje conforme aumenta la edad, con diferencias significativas en el conocimiento de acuerdo a la escolaridad, observaron una tendencia significativa $(\mathrm{p}=0.000)$ entre mayor escolaridad mayor conocimiento de métodos en jóvenes de 15 a 19 años de edad.

En relación con el inicio de vida sexual, encontraron que el $16.4 \%$ la habían iniciado, en el grupo de edad de 18 a 19 años de sexo masculino con un valor de $\mathrm{p}=0.0028$ y de estos solo $37 \%$ usaron algún método anticonceptivo en la primera relación sexual, señalando que el sexo masculino de mayor escolaridad, con información de algún método anticonceptivo y con actividad sexual a una edad mayor, tuvieron mas probabilidad de usar anticonceptivos en su primera relación sexual, por lo que concluyen que la población adolescente que ha tenido relaciones sexuales no utilizo métodos anticonceptivos durante su primera relación sexual, lo cual incrementa la posibilidad de embarazos.
Vega de la Cruz et $\boldsymbol{a l}^{7}$ realizaron un estudio descriptivo, Observacional, transversal y prospectivo en población de 10 a 19 años, con el propósito de identificar inicio de vida sexual por género, encontrando que de las mujeres $48.20 \%$ tienen actividad sexual, y de estas el 28.72\% comenzaron antes de los 15 años y el $71.27 \%$ después de los 15 años; de los hombres el 59.48\% refirió tener relaciones sexuales ; el $13.79 \%$ antes de los 15 años y el 86.20\% después de los 15 años.

Los autores concluyen que la actividad sexual de los adolescentes, en la mujer es más temprana, sin embargo es menor en relación con el hombre adolescente, ya que este una vez que la inicia, presenta más continuidad en su actividad sexual que la mujer.

Por otra parte Elías García y cols. ${ }^{8}$ realizaron un estudio con el propósito de caracterizar algunos aspectos de la sexualidad en un grupo de adolescentes de 12 a 19 años en el área de salud del Policlínico Victoria Cuba-Angola, mediante un estudio no experimental de tipo descriptivo y de corte transversal.

Los autores señalan que del total de adolescentes (102) el 61.7\% había tenido relaciones sexuales, destacándose el sexo masculino con un $66.7 \%$, y el femenino con mas de la mitad (56.2\%)

El 69.8\% de los jóvenes estudiados tuvieron relaciones sexuales antes de los 15 años, siendo el sexo masculino el de mayor porcentaje, con un $86.2 \%$. En el caso de las mujeres observaron que el $51.8 \%$ tuvo su primera relación sexual después de los 15 años; el método anticonceptivo mas utilizado fue el condón. 
Las fuentes de información y orientación sobre sexualidad y sexo el 95\% refirió que con los amigos; el 64.4\% la escuela; $58.8 \%$ los padres; los hermanos mayores $56.8 \%$ y con la pareja el $50 \%$.

En lo que respecta al numero de parejas sexuales el $66.7 \%$ tenia una sola pareja al momento del estudio, aunque el sexo masculino se evidencio que mas del $50 \%$ se relacionó con 2 o mas parejas.

Los autores concluyen que los adolescentes comenzaron sus relaciones sexuales antes de los 15 años, predomino la inestabilidad de la pareja y el método anticonceptivo mas utilizado fue el condón.

Ospina et $\mathrm{al}^{9}$ realizaron un estudio de corte transversal con el propósito de describir prácticas y comportamientos sexuales en 764 estudiantes universitarios.

La edad promedio fue de 20.45 años, predominando el sexo femenino $50.7 \%$, y el $55.2 \%$ con relación sexo afectiva estable.

El $87.8 \%$ de hombres y $67.8 \%$ de mujeres con vida sexual, la edad promedio de inicio 15.5 años en hombres y 17.5 años en mujeres, con un promedio de compañeros sexuales 3.96 en hombres y 1.91 en mujeres.

El método anticonceptivo mas conocido es el condón $56.6 \%$ referido por hombres y mujeres; además de ser el método mas utilizado $22.4 \%$.

Los autores concluyen que los factores de riesgo identificados en la salud reproductiva es el bajo nivel de conocimiento sobre sexualidad y anticoncepción.

En la revisión de la literatura sobre anticoncepción se ha reportado que poco mas de la mitad de adolescentes que tienen relaciones sexuales usan métodos anticonceptivos, siendo el mas común el condón ${ }^{4}$, la otra mitad de adolescentes con vida sexual activa que no utilizan métodos anticonceptivos, las razones mencionadas por ellos es por desconocimiento de los métodos y/o sus formas adecuadas de uso $^{10}$, creencias negativas asociadas con su uso ${ }^{11}$ y la falta de planeación en las relaciones sexuales ${ }^{12}$

Con base a lo anterior, esta temática se convierte así en un punto es de gran relevancia para el profesional de enfermería en la generación de conocimiento sobre el comportamiento sexual de los adolescentes para desarrollar modelos educativos basados en evidencia científica.

El propósito de esta investigación fue describir las características de la conducta sexual de los adolescentes, variables que se relacionan con el inicio de la actividad sexual y prácticas de riesgo para embarazo, en $\mathrm{Cd}$. Victoria, Tamaulipas 2008.

\section{Metodología}

Tipo de estudio: Observacional, descriptivo, transversal, coorelacional $^{2}$

Población, muestra y muestreo: 1212 Adolescentes de 12 a 19 años ambos sexos estudiantes de 6 Escuelas Secundarias Generales y 2 Preparatorias Federales de Cd. Victoria, Tam. Muestreo no probabilístico, por disponibilidad.

Instrumentos de Recolección de Datos: Se elaboro un instrumento ex profeso integrado por tres secciones; una Cédula con datos sociodemográficos conformada por 8 preguntas abiertas; en la segunda sección se indaga sobre conocimiento de métodos anti- conceptivos dos preguntas dicotómicas y 2 de selección múltiple; la tercera sección corresponde a la actividad sexual 10 preguntas 7 abiertas y 3 dicotómicas, se implemento la prueba piloto con el propósito de aplicar el instrumento, y detectar posibles problemas que puedan surgir mediante la obtención de la información, así como el tiempo empleado obteniéndose una confiabilidad del instrumento de un alpha de cronbach de 0.82 .

\section{Definición de VARIables}

Comportamiento sexual: Vía de procreación y, lo que les resulta mas importante, como via de disfrute sexual y de atracción física erótica para otras personas, sus manifestaciones pueden clasificarse en fantasías y deseos, caricias, atracción y búsqueda de pareja, y coito ${ }^{6}$ Esta variable se midió através de la edad de inicio de relaciones sexuales y número de parejas.

Factores de riesgo: Características individuales e interpersonales que presenta el adolescente que puede incrementar la probabilidad de iniciar la vida sexual sin protección; se midió mediante la edad de inicio de vida sexual, el nivel de conocimiento sobre métodos anticonceptivos y su uso en la primera relación sexual.

Los datos fueron procesados através del programa estadístico Statical Package for the Social Sciences (SPSS) version 16.0 para Windows. Se utilizó la estadística descriptiva, medidas de tendencia central y tablas de contingencia para la correlación de las variables.

Posterior a la autorización de los padres o tutores, se procedió a la aplicación del cuestionario a estudiantes que presenten el consen- 
timiento por escrito de los padres así como de ellos mismos, en un lugar privado asignado por los directivos de las escuelas.

El estudio fue aprobado por el Comité de Ética e Investigación de la Facultad de Enfermería de la Universidad Autónoma de Tamaulipas (UAT) y la autorización por escrito a las autoridades de las instituciones la Secretaría de Educación Pública así mismo el consentimiento informado firmado por el padre o tutor o el mismo estudiante.

\section{Resultados}

Los adolescentes estudiados provienen de 6 escuelas secundarias (746) y dos Preparatorias (466), distribuidos equitativamente entre turnos, 609 y 603 matutino y vespertino respectivamente. En relación al género las mujeres tienen una mayor representación, 54\% del total. La mitad de los casos se encuentra en el rango de 15 a 17 años, mientras que los casos entre 18 y 19 años solo representan un 6\% del total.

Respecto al estado civil, solo el $2 \%$ de los casos están en unión libre, de los cuales la mayoría son mujeres, no hay relación con la edad. El resto de los entrevistados son solteros, y como es de esperarse para este grupo de edad la mayoría vive con sus padres o familiares (98.5\%), solo $1.5 \%$ vive con amigos u otras personas. No hay relación clara entre los integrantes de la vivienda y la edad, el género o si el individuo se encuentra en unión libre o no.

Por otro lado, la mayoría de los participantes tienen acceso a servicios de salud, que no está asociado ni al género ni a edad de los individuos; un $21 \%$ de ellos refieren ingreso mensual menor a tres salarios mínimos. (\$4500.00). Al discrimi- nar por escuela se puede ver que este porcentaje va desde $6 \%$ para la Secundaria Gral. No. 7 hasta 37\% para la Secundaria Gral. No. 5, lo que representa un nivel socioeconómico bajo para los estudiantes de la Secundaria No.5

En lo que respecta a la información y conocimiento sobre sexualidad, el 97\% tienen información sexual, la principal vía de información fue la escuela y los amigos. Llama la atención que entre quienes dicen conocer sobre métodos anticonceptivos el 38\% dice no conocer su funcionamiento y solo el 34\% de los casos estudiados dicen conocer el funcionamiento del método anticonceptivo. Ante la pregunta ¿cuándo es más probable que una mujer se embarace?, la respuesta más frecuente fue "no se", entre las mujeres parece haber un mayor conocimiento que entre los hombres, con un 14 y $10 \%$ de respuestas correctas.

No existe mayor consistencia entre el conocimiento de los métodos anticonceptivos y de su funcionamiento, con conocer el momento de mayor probabilidad de embarazo. A pesar de que un $62 \%$ de casos responden que conocen sobre los métodos y su funcionamiento, solo un $12 \%$ sabe cuándo es el momento de mayor fertilidad.

En relación a la actividad sexual y uso de anticonceptivos, el 22\% de los entrevistados tuvieron actividad sexual, la mayoría iniciándose a la edad de 14-15 años (47\%).

Poco más de la mitad de quienes iniciaron su vida sexual usó algún método anticonceptivo en su primera relación. Quienes iniciaron más temprano su vida sexual, 12 a 15 años, presentan un porcentaje menor de uso de método anti-

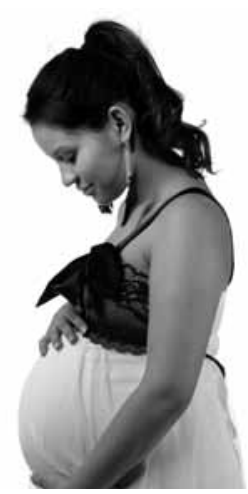

conceptivo en su primer relación, mientras que quienes iniciaron entre los 16 y 19 años utilizaron en mayor porcentaje algún método anticonceptivo.

Solo un 36\% utilizan algún método anticonceptivo de manera esporádica y los pocos casos con más tiempo de uso de anticonceptivos están relacionados con la edad de la población estudiada; los motivos por no usar método anticonceptivo, la mayor parte dice que se debe a no planear la relación sexual y un $7 \%$ porque deseaba embarazarse; a pesar de que las opciones de respuesta son distintas para la primera y la última relación sexual, estos porcentajes resultan similares, siendo la primer causa de no uso de método anticonceptivo el no planear la relación sexual.

Al analizar el uso de método anticonceptivo tanto en la primera como en la última relación sexual, no se encontró asociación con ninguna de las variables consideradas que fueron: edad, género, grado (secundaria y bachillerato), información sobre sexualidad, conocimientos sobre métodos anticonceptivos y conocimiento sobre el funcionamiento de los métodos anticonceptivos.

Todos los efectos tuvieron un valor de p mayor a 0.176 , se utilizaron las mismas variables para explicar el embarazo y ninguna resulto significativa. 
$\mathrm{Al}$ analizar la última relación sexual y el número de parejas, la mitad de los casos tiene más de una pareja, no se puede separar entre 1 o 2 parejas ya que esta es una categoría. La gran mayoría ha tenido actividad recientemente (76\%) que es entre una semana y un mes en que se tuvo la última relación sexual, quienes tienen más de 4 parejas, presentaron actividad sexual más recientemente, de estos los hombres tienen en mayor porcentaje más parejas. No hay diferencias en cuanto al momento de la última relación sexual entre hombres y mujeres, tampoco se identifica una asociación entre la edad y el número de parejas, ni entre la edad y la última vez en que se tuvo relación sexual.

Por otro lado, se analizo el inicio de la vida sexual en función de la edad, género y grado; para el análisis se considera la pertenencia a secundaria o bachillerato y no se tomaron en cuenta los individuos en los grupos de 18 a 19 años en secundaria y de 12 a 14 años en bachillerato. El efecto de la edad se estudio anidado en grado y no se encontró interacción entre género y edad, obteniéndose el mismo comportamiento respecto a la edad para ambos géneros. Las tres variables resultaron significativas ( $p>0.01)$ en su efecto principal. El efecto de la edad se considero anidado en grado debido a la relación existente entre estas variables.

En la tabla 2 se presentan los porcentajes estimados en cada una de las categorías de estas variables, las mujeres presentan un mayor porcentaje de casos sin vida sexual que los hombres, en secundaria hay una mayor porcentaje de casos sin inicio de actividad sexual, mientras que a mayor edad hay un mayor inicio de actividad sexual, tanto en secundaria como en bachillerato; si se compara el bachillerato contra la secundaria solo en la edad común de 15 a 17 años, vemos que en secundaria hay menor porcentaje de individuos que no iniciaron su vida sexual. Esto puede indicar que más allá de la edad, que es un factor para el inicio de la actividad sexual, también hay un efecto del entorno del estudio.

$\mathrm{Al}$ analizar la edad de inicio de vida sexual, nos encontramos nuevamente con las mismas variables explicativas significativas, genero, grado y edad anidada en grado $(\mathrm{p}<0.04)$, no encontrándose tampoco efecto de las interacciones.

\section{Discusión}

La información obtenida en nuestro estudio es similar a trabajos previos Encuesta Nacional de la Juventud",
(16 años) Elías y cols ${ }^{8}$,(15 años), Ospina et al ${ }^{9}$, donde se refleja que la edad de inicio de vida sexual es en el rango de 14 a 15 años, el grupo de secundaria muestra una tendencia a iniciar la actividad sexual más temprano comparado con el grupo de bachillerato, al discriminar por género los hombres tienden a iniciar la vida sexual más temprano que las mujeres, no utilizando método anticonceptivo en su primera relación sexual.

En relación al conocimiento sobre métodos anticonceptivos, su alcance es limitado, la principal fuente de información lo constituyen la escuela y los amigos; el 97\% de los participantes dice conocer métodos anticonceptivos y solo el 34\% refirió conocer su funcionamiento.

Estos resultados dejan ver la necesidad de diseñar estrategias educativas para este grupo de población sobre la temática de salud sexual y

\section{TABLA 2.}

\section{Porcentajes estimados de inicio de actividad sexual} según género, grado y edad dentro de grado.

\begin{tabular}{|c|c|c|c|c|c|}
\hline & & & \multicolumn{3}{|c|}{$\begin{array}{l}\text { Porcentaje de individuos } \\
\text { con actividad sexual }\end{array}$} \\
\hline & & & $\begin{array}{c}\text { Límite } \\
\text { inferior } \\
(95 \%)\end{array}$ & Media & $\begin{array}{c}\text { Límite } \\
\text { superior } \\
(95 \%) \\
\end{array}$ \\
\hline \multirow{2}{*}{ 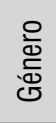 } & M & & 77 & 81 & 85 \\
\hline & $\mathrm{H}$ & & 58 & 63 & 68 \\
\hline \multirow{2}{*}{ 票 } & Secundaria & & 83 & 86 & 89 \\
\hline & Bachillerato & & 47 & 54 & 61 \\
\hline \multirow{4}{*}{ 宽 } & \multirow{2}{*}{ Secundaria } & $12-14$ & 88 & 91 & 93 \\
\hline & & $15-17$ & 75 & 81 & 85 \\
\hline & \multirow{2}{*}{ Bachillerato } & $15-17$ & 63 & 68 & 72 \\
\hline & & $18-19$ & 28 & 39 & 53 \\
\hline
\end{tabular}


reproductiva es indispensable considerar que las causas de indicio de actividad sexual y embarazo a temprana edad, es multifactorial.

\section{Conclusiones.}

Las características de los individuos incluidos en el estudio corresponden a una población adolescente predominantemente urbana de escuelas públicas que aún viven con sus padres y con una relación de géneros acorde a la población general.

En relación al propósito del estudio relacionado a la conducta sexual de los adolescentes y, las variables que se relacionan con el inicio de la actividad sexual y riesgo de embarazo, se identifico que la actividad sexual inicia más tempranamente en los hombres que en las mujeres. El uso de anticonceptivos no es generalizado ni en la primera ni en la última relación sexual, siendo el motivo principal de no usarlo el tener relaciones sexuales no planeadas.

El conocimiento sobre el ciclo reproductivo femenino no es muy bueno tampoco, aún entre quienes dicen conocer sobre métodos anticonceptivos y su funcionamiento la principal vía de información sobre sexo es la escuela y los amigos, no pudiéndose discriminar cual es el mecanismo escolar para obtener información sexual, al respecto una limitante que presenta el estudio es que no se midió la calidad la educación sobre esta temática que tienen los adolescentes.

A manera de conclusión, este estudio refleja un panorama general del comportamiento sexual de los adolescentes de Cd. Victoria, Tamaulipas, con resultados similares a otras investigaciones realizadas en México y otros países latinoamericanos, lo que pone de manifiesto la necesidad de realizar estudios de intervención educativa para la prevención de conductas sexuales de riesgo, donde el profesional de enfermería constituye un grupo imprescindible en la educación sanitaria

\section{Agradecimiento.}

A la Universidad Autónoma de Tamaulipas, por el apoyo Institucional brindado para la realización de esta investigación.

\section{Referencias Bibliográficas}

1 Sistema de Información en Salud (SIS) Jurisdicción Sanitaria No. 1. Departamento de Estadística Enero-Diciembre. 2008 Cd. Victoria, Tamaulipas.

2 Polit, F. \& Hungler, P. (1999). Investigación científica en ciencias de la salud (6a. ed.) México, DF: McGraw-Hill Interamericana.

3 Organización Mundial de la Salud [OMS] (1999). Programación para la Salud y el Desarrollo de los Adolescentes. Ginebra: OMS; 1999. Serie de Informes Técnicos, 886. Disponible en: http://www.paho.org

4. Encuesta Nacional de la Juventud (2000) Resultados Generales. México DF. Instituto Nacional de la Juventud.

5 Organización Mundial de la Salud \& Fondo de Población de naciones Unidas (2000) "Doble protección contra las infecciones de transmisión sexual incluyendo el SIDA y el embarazo no deseado". Ginebra: Organización Mundial de la Salud. Programa de Naciones Unidas sobre VIH/SIDA y Fondo de Población de naciones Unidas

6 González GC, Hernández SA. Oláis FG. "Perfil del comportamiento sexual en adolescentes de 12 a
19 años de edad: resultados de la ENSA 2000". Salud Pública de México. Cuernavaca Vol. 47 No. 3. Junio 2005.

7 Vega - De La Cruz Fermín Gerson, Gómez Soto Víctor, "Inicio de la vida sexual en el adolescente", VII Foro Delegacional de la Investigación en Salud del IMSS en Nuevo León (México), Edición especial. No .2-2003.

8 Elías García Y,; Guridi González M., Dorta Guridi Z., Noda L., Reyes Díaz Z. Comportamiento de la sexualidad en un grupo de adolescentes del área de salud de Mulgoba. Revista Electrónica PsicologiaCientifica.com ISSN: 2011-2521. Disponible en: http://www.psicologiacientifica.com/bv/index.php consultada: 10 Nov. 2006

9 Ospina Díaz J.M., Manrique-Abril F.G. Practicas y comportamientos sexuales en estudiantes universitarios. Revista Avances en Enfermería V. 25 No.2 Bogota Jul/Dic. 2007. versión On -line. Disponible en: http://www.enfermeria.unal. edu.co/revista/

10 Tapia-Aguirre V., Arillo- Santillán E., Allen B., Ángeles Llerena A., Cruz- Valdez A., \& Lazcano-Ponce E. (2004) "Associations among condom use, sexual behavior, and knowledge about HIV/ AIDS : A study of 12, 393 public school students. Archives of Medical Research " 35, 334-343

11 Lopez M. (2000) La personalidad y su relación con la percepción de riesgo en relaciona al SIDA. Tesis de Maestría no publicada. Universidad Nacional Autónoma de México.

12 Neechi S. \& Schufer M. (1999) "Iniciación sexual y anticoncepción en el adolescente varón de la Ciudad de Buenos Aires", Archivos Argentinos de Pediatría 97 (2) 101-108 